\title{
Understanding and Managing Pain Patients
}

\author{
Kavitha Prasad* \\ Department of Oral and Maxillofacial Surgery, India
}

ISSN: 2637-7764

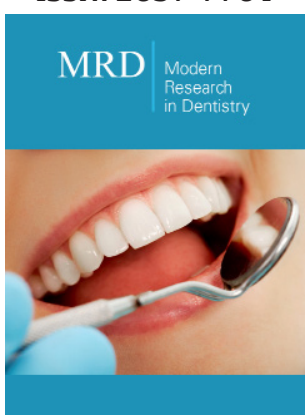

${ }^{* 1}$ Corresponding author: Kavitha Prasad, Department of Oral and Maxillofacial Surgery

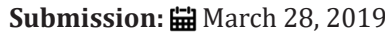

Published: 俩April 04, 2019

Volume 3 - Issue 5

How to cite this article: Kavitha P. Understanding and Managing Pain Patients. Mod Res Dent. 3(5) MRD.000573.2019.

DOI: 10.31031/MRD.2019.03.000573

Copyright@ Kavitha P, This article is distributed under the terms of the Creative Commons Attribution 4.0 International License, which permits unrestricted use and redistribution provided that the original author and source are credited.
Opinion

It requires a certain quality in the treating doctor to actually listen to the patients pain experience, connect with the patient and help him or her to deal with the pain in the best possible way. Understanding pain is not easy, as it is a very subjective, individualized feeling which only the person experiencing it is in complete touch with. Many times they are unable to completely describe their pain in words. It is something which is experienced and can never be fully explained. What one experiences is multi-dimensional with a complex mix of psychological, emotional, behavioral and lifestyle traits of the individual. The actual pain the patient perceives may be multiplied many times due to these factors. Pain which most of us understand is when it is associated with some pathology, condition, disease or injury which when treated; pain gradually subsides with the patient returning to near normalcy. The real challenge is when we cannot exactly diagnose the cause for the patient's pain and in spite of symptomatic and supportive treatment, patient still complains of pain, inability to attend to normal daily activities and a feeling of helplessness. These patients consult with multiple doctors in search of a remedy.

During the process, they may have undergone various investigations; beginning from routine tests to advanced immunological and imaging tests in search of the cause. Following this, treatment is again symptomatic and supportive. Sometimes, some surgical interventions are also carried out; removal of teeth in the area of pain, removal of deeply impacted teeth, arthroscopy and condylar shaving, lavage of salivary glands etc. in the hope that the patient gets some relief from pain. Physiotherapy in the form of TENS, shortwave diathermy, ultrasound therapy and exercises are advised. Analgesics are given; to begin with NSAIDS followed by centrally acting and opioid derivatives in more severe pain. Exercises, Vitamin and mineral supplements, diet and lifestyle modifications are some of the supportive treatments offered. Counseling the patients is a very important aspect which most of the times is not adequately done. One must make time to actually listen to the patients complaints at length, be attentive and empathetic to the patients sharing their pain experience. Patients don't reveal many of things which are bothering them if they feel the doctor is casual and not taking what they are saying seriously. Helping the patients to help themselves is the only way out in chronic pain patients. One can do that by being a good listener and creating an environment of comfort and warmth so that they can come out with all their bottled emotions in the context of their pain. When the patient feels that someone understands what they are going through and are considerate to their feelings and respond appropriately, healing begins. Slowly the doctor should make the patient understand that it is in the patient's own hands how he accepts his situation, his pain and help them to work out a management strategy, together, in consensus. Every individual seeks to be loved, wanted and respected. When they don't feel fulfilled, they unconsciously create situations and symptoms which will cause them pain, either physical or mental to draw attention and love.

When the patient's confidence is won, he will be more receptive to the treatment, relief from pain will eventually follow. Alternate therapies such as hypnosis, acupressure, yoga, ayurveda and homeopathic treatments can be incorporated, depending on patient preference and compliance for their wholesome and long-term well-being. Integrated treatment is the future and a reality when it comes to treating pain and other conditions where no one type 
of medicine can offer complete cure. Integrated Medicine should be practiced and protocols set in place for pain management; this will prevent the patient from seeking multiple doctors and centers in the hope of getting a solution. Over a period of time, patients realize that nobody can help them as well as they can help themselves.
They learn what works for them and what does not. The moment they take responsibility of their lives, they begin to feel and do better. What is required of the doctors is to be understanding and supportive and help the patients overcome their pain situations.

For possible submissions Click below: 